\title{
Interaction of galaxies with the intra-cluster medium and ICM metal enrichment
}

\author{
Sabine Schindler ${ }^{* \dagger}$ \\ University of Innsbruck, Institute of Astrophysics, Austria \\ E-mail: sabine.schindler@uibk.ac.at
}

\begin{abstract}
Interaction processes between the components of clusters of galaxies are efficient in removing gas from the galaxies and transporting it into the intra-cluster medium. This transport is responsible for the high metallicities found in the intra-cluster medium. So far it is not quite clear what the efficiencies of the various processes and their time dependencies are. The current status of observational evidence in terms of metallicity measurements in clusters, spatial metallicity distribution and metallicity evolution is reviewed in this article. Different types of interaction and metal enrichment processes are discussed: galactic winds, ram-pressure stripping, galaxy-galaxy interaction, jets from AGN, and an intra-cluster stellar population. Results of simulations are presented which take some of these interaction processes into account.
\end{abstract}

Baryons in Dark Matter Halos

5-9 October 2004

Novigrad, Croatia

\footnotetext{
${ }^{*}$ Speaker.

${ }^{\dagger}$ A footnote may follow.
} 


\section{Introduction}

The components of clusters of galaxies - galaxies and intra-cluster medium (ICM) - interact with each other in many ways. There is more and more observational evidence that various types of interaction processes are at work, which remove interstellar medium (ISM) from the galaxies. This removed ISM intermixes with the ICM, so that the nowadays observed ICM is a mixture of both.

The deep gravitational potential wells of clusters retain the integrated products of nucleosynthesis. With the new X-ray observations metals in the intra-cluster gas can be measured in detail. As the intra-cluster gas represents most of the baryonic matter these X-ray observations are of high importance for the understanding of the evolution of clusters and their galaxies. Most of the metals must have been produced within the galaxies and later they have been transported by various interaction processes from the galaxies into the intra-cluster gas. Hence the metals are a good tracer for the interaction processes between galaxies and the intra-cluster gas.

Previous X-ray observations have shown already that the metal abundance in the intra-cluster medium is quite high, about one third in solar units (e.g. Fukazawa et al. 1998 (ASCA), Peterson et al. 2003 (XMM), De Grandi et al. 2004 (BeppoSAX)) and already many years ago there have been attempts to infer the origin of the metals (Arnaud et al. 1992). With the old X-ray satellites ROSAT, ASCA, and BeppoSAX, however, it was hard to draw conclusions on the metal distribution any more detailed than a radial metallicity profile (see e.g. De Grandi et al. 2004) and on the evolution of metals with redshift (Schindler et al. 1999).

The new generation of X-ray satellites XMM and CHANDRA brought a break through in the investigation of the metal enrichment. Their superior spatial and spectral resolution together with the high sensitivity made it possible to measure 2D distributions of metals: metallicity maps (e.g. Schmidt et al. 2002; Furusho et al. 2003; Sanders et al. 2004; Fukazawa et al. 2004; Hayakawa et al. 2004). These maps shows that, the metallicity has clearly a non-uniform distribution and a non-spherical distribution, which leads to very interesting conclusions. The maps explain why the metallicity profiles, in which all metallicities are azimuthally averaged, show a flat distribution in non-cooling cores clusters: due to the accumulation of photons from regions very far apart all variations are smeared out.

Further information could be provided by observations of metals in distant clusters. So far attempts have been made to measure metallicity evolution out to a redshift of 1.2 (Tozzi et al. 2003), but the situation is not very clear yet (Nagashima et al. 2004). Different distributions of Fe and other elements can be observed (e.g. Peterson et al. 2003; Tamura et al. 2004), which allow for conclusions on the contributions of the different types of supernovae.

Together with the metals also energy is transported into the ICM. This topic has received a lot of attention recently because it is considered a possible solution for the cooling/heating balance in cooling core clusters (see Churazov et al. 2002; Böhringer et al. 2004; Fabian 2004).

\section{Metal enrichment processes}

All these new results have stimulated a lot of discussion on the origin of metals and the energy transfer. Given that most metals must come from cluster galaxies, the high ICM metallicity $(0.3$ 
solar) and the small contribution of the galaxy masses to the baryonic matter in a cluster $(\approx 20 \%)$ the amount of material that has to be transported from the ISM to the ICM must be considerable.

\subsection{Galactic winds}

Already many years ago galactic winds were suggested as a possible ISM transfer mechanism (De Young 1978). The winds are driven by supernova explosions. Since then winds were often held responsible for most of the gas transport. But recent observations and simulations indicate that other processes can contribute considerably.

\subsection{Ram-pressure stripping}

One process that obtains more and more attention is ram-pressure stripping (Gunn \& Gott 1972). In the Virgo cluster alone at least 7 examples of ram-pressure affected spiral galaxies have been found by HI observations (Cayatte et al. 1990; Veilleux et al. 1999, Vollmer et al. 1999, Vollmer 2003, Kenney et al. 2004, Vollmer et al. 2004a,b; Koopmann \& Kenney 2004) and in elliptical galaxies stripping features have been discovered in X-ray observations (e.g. Rangarajan et al. 1995). Also in the Coma and other clusters evidence for ram-pressure stripping has been found (Bravo-Alfaro et al. 2000, 2001).

\subsection{Galaxy-galaxy interaction}

Another possible mechanism to remove gas from the galaxies is interaction between the galaxies (e.g. Clemens et al. 2000). While the direct stripping effect is probably not very efficient in clusters due to the short interaction times, the close passage of another galaxy can trigger a star burst (Barnes \& Hernquist 1992; Moore et al. 1996; Bekki 1999), which subsequently can lead to a galactic wind (Kapferer et al., in prep.). Star bursts with subsequent winds can also be caused by cluster mergers (Ferrari et al. 2003,2004), because in such mergers the gas is compressed and shock waves and cold fronts are produced (Evrard 1991; Caldwell et al. 1993; Wang et al. 1997; Owen et al. 1999; Moss \& Whittle 2000; Bekki \& Couch 2003). But there can also be a competing effect. The ISM might be stripped off immediately by ram-pressure stripping (Fujita et al. 1999, Heinz et al. 2003) and hence the star formation rate could drop. In any case ISM would be removed from the galaxies.

\subsection{Jets from AGN}

Jets from AGN interact with the ICM (e.g. Schindler et al. 2001, Blanton et al. 2001, McNamara et al. 2001, Heinz et al. 2002, Choi et al. 2004). As this jet-ICM interaction can have an effect both on the energetics and the metal enrichment of the ICM several groups have started to calculate also this process (Zhang 1999, Churazov et al. 2001, Nulsen et al. 2002, Krause \& Camenzind 2003, Heinz 2003, Beall et al. 2004, Della Vecchia et al. 2004).

\subsection{Intra-cluster stellar population}

There is more and more evidence for a population of stars between the galaxies in a cluster (Gerhard et al. 2002; Cortese 2004; Ryan-Weber et al. 2004; Adami et al. 2004). When these stars explode as supernovae they can enrich the ICM very efficiently (Domainko et al. 2004a; Zaritzsky 
et al. 2004; Lin \& Mohr 2004), therefore this population of stars should also be considered for the enrichment processes.

\section{Simulations}

Obviously the enrichment of the ICM is a complex process, which is the result of many different mechanisms. Many groups have done already some time ago simulations to quantify one or more of these enrichment mechanisms: winds (David et al. 1991; Metzler \& Evrard 1994, 1997) and winds compared to galaxy-galaxy interactions (Gnedin 1998; Aguirre et al. 2001). These old simulations came to very different conclusions. A problem with this kind of simulations was that they had to cover large scales as well as galaxy scales. Therefore the resolution was not very good at small scales and hence the results had large uncertainties, which were probably the reason for the discordant results.

In order to overcome the problem of the large range of scales several groups simulated in detail a single galaxy, which is in the process of being stripped (Abadi et al. 1999; Quilis et al. 2000; Toniazzo \& Schindler 2001; Schulz \& Struck 2001; Vollmer et al. 2001; Hidaka \& Sofue 2002; Bekki \& Couch 2002; Otmianowska-Mazur \& Vollmer 2003; Schumacher \& Hensler 2004). From these simulations of spirals and ellipticals the efficiency and time scales of the stripping process depending on galaxy properties and on ICM conditions can be inferred.

Currently, several approaches are being made to explain the overall enrichment of the ICM. De Lucia et al. (2004) and Nagashima et al. (2004) use a combination of semi-analytic techniques and N-body simulations to calculate the overall ICM metallicity, i.e. they do not predict the distribution of metals in a cluster. They find that mainly the massive galaxies contribute to the enrichment and that there is a mild metal evolution since $z=1$. Tornatore et al. (2004) and Valdarnini (2003) do hydrodynamic simulations with smoothed particle hydrodynamics that include detailed yields from type Ia and II supernovae, but do not distinguish between the different transport processes. They put most of their emphasis on the amount of iron produced and on profiles. Tornatore et al. find interesting results among them profiles that are significantly steeper than those observed. Springel \& Hernquist (2003) use a multi-phase model and include also some enrichment processes.

The Hydro SKI Team in Innsbruck takes a complementary approach. We investigate the transport of the metals from the galaxies into the intra-cluster medium and study in detail the efficiency of the various transport processes, which are neglected by the other groups. In the first simulations, that we have performed so far, we have taken into account two different transport processes - rampressure stripping and galactic winds (see http://astro.uibk.ac.at/astroneu/hydroskiteam/index.htm). First preliminary results and techniques have been shown in the contributions by W. Kapferer, W. Domainko and T. Kronberger (see these proceedings) and have been published in Domainko et al. (2004b,c,d,e); Kapferer et al. (2004a,b); Mair (2004); Schindler et al. (2004).

From these simulations we can draw some preliminary qualitative conclusions for the comparison of the enrichment efficiency of ram-pressure stripping and galactic winds (see Fig. 1). We find that ram-pressure stripping is definitely important for the enrichment of the ICM, in contrast to what has been claimed frequently in the past. We also find that the distribution of metals coming from the two processes is different. Ram-pressure stripping is more efficient in the centre of the cluster. The reason is that the stripping rate is proportional to the density of the ICM and propor- 


\section{Metallicity map}

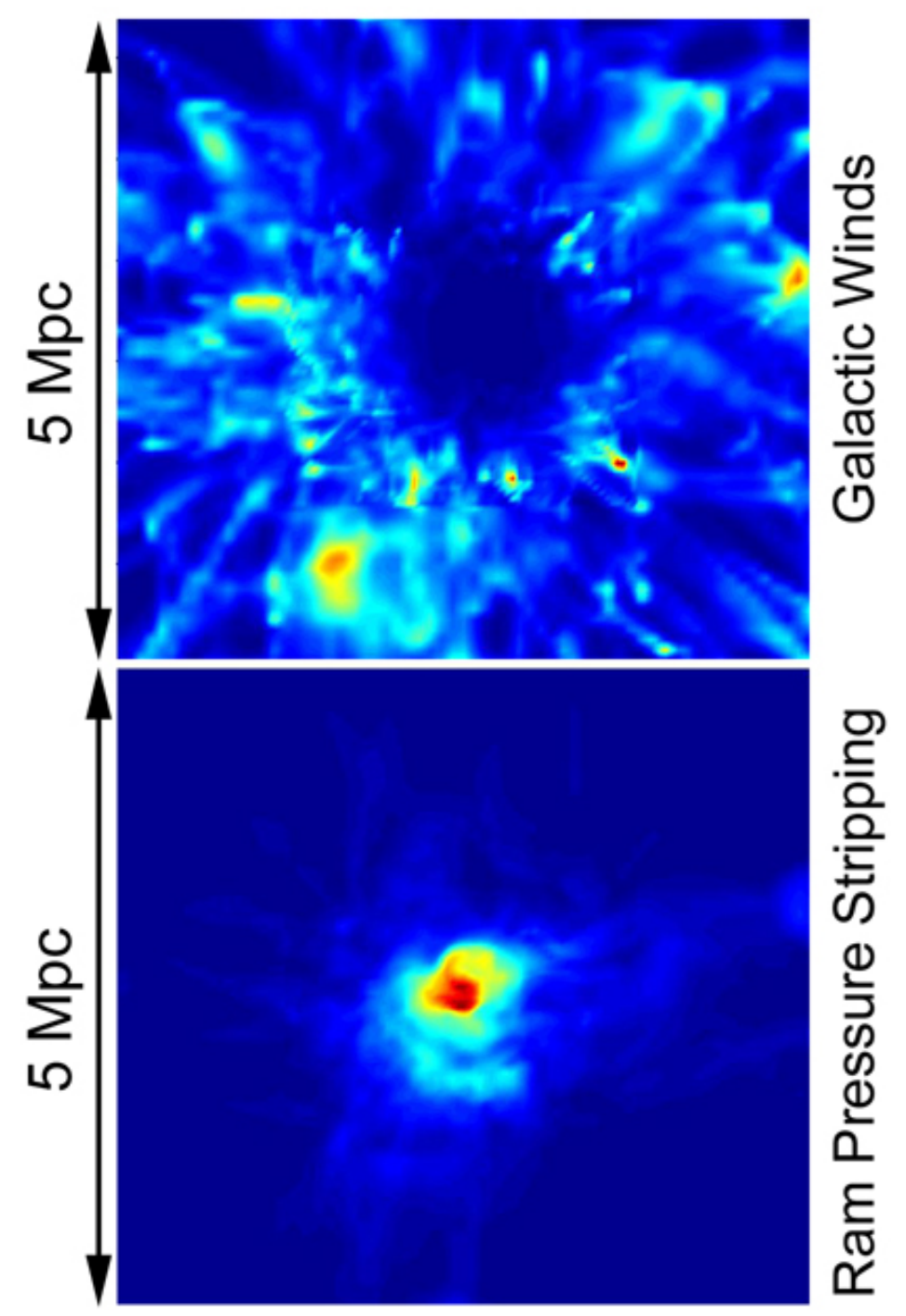

Figure 1: Metallicity distribution in a simulation of a massive, Coma-type cluster. Top: The simulation takes galactic winds into account. The metallicity shows a minimum in the cluster centre, because the ICM pressure in such a massive cluster is so strong that it suppresses winds here. Bottom: In this simulation only the effect of ram-pressure stripping is included. The metallicity distribution is concentrated towards the cluster centre, because stripping is most efficient in the centre.

tional to the square of the velocity of the galaxies. Both ICM density and galaxy velocity increase towards the cluster centre, therefore more gas is lost there. For galactic winds the metallicity maps look very different: in the cluster centre not a lot of ISM is lost due to winds. Again the explanation is clear: in the central parts the ICM pressure is higher therefore a possible galactic wind feels more pressure from outside, which might prevent it from blowing gas away. We find that the mean radius of pressure balance between ICM and galactic wind depends strongly on the dynamical state of the cluster: for a relaxed Coma-like cluster and a Galaxy-like spiral the mean radius of pres- 
sure balance is at about $0.8 \mathrm{Mpc}$, while for a merger in the same cluster the radius is at about 1.2 Mpc. Interesting is also that the different distributions are not dispersed immediately by the motion of the ICM, but they are relatively stable over some Gyrs. This inhomogeneity in the metallicity distribution is on good agreement with recent X-ray observations.

\section{Ackowledgements}

I thank the Hydro SKI Team and the collaborators Maximilian Ruffert and Dieter Breitschwerdt for the very pleasant and fruitful cooperation. This work is supported by the Austrian Science Foundation FWF under grant P15868 and by the UniInfrastructure Programme 2004 by the Austrian Ministry for Education, Science and Culture.

\section{References}

Abadi, M.G., Moore, B., Bower, R.G., 1999, MNRAS, 308, 947

Adami C., Slezak E., Durret F. et al. 2004, astro-ph/0409108

Aguirre, A., Hernquist, L., Schaye, J., Katz, N., Weinberg, D.H., et al. 2001, ApJ, 561, 521

Arnaud M., et al. 1992 A\&A 254, 49

Barnes, J., \& Hernquist, L., 1992, A\&A, 30, 705

Beall J.H., Guillory J., Rose D.R., Schindler S., Colafrancesco S., 2004, Chin. J. Astron. Astrophys., in press

Bekki, K., 1999, ApJL, 510, 15

Bekki K., Couch W., 2002, ApJ 577, 651

Bekki K., Couch W., 2003, ApJ 596, L13

Blanton E.L., Sarazin C.L., McNamara B.R., Wise M.W., 2001 ApJ 558, 15

Böhringer, H., Matsushita, K., Churazov, E., Finoguenov, A., 2004, in: The Riddle of Cooling Flows in Galaxies and Clusters of Galaxies, Eds. T. Reiprich, J. Kempner, and N. Soker.

Bravo-Alfaro H., Cayatte V., van Gorkom J.H., Balkowski C., 2000, AJ, 119, 580

Bravo-Alfaro H., Cayatte V., van Gorkom J.H., Balkowski C., 2001, A\&A 379, 347

Caldwell, N., Rose, J.A., Sharples, R.M., Ellis, R.S., Bower, R.G., 1993, AJ, 106, 473

Cayatte V., van Gorkom J.H., Balkowski C., Kotanyi C., 1990, AJ, 100, 604

Choi Y., Reynolds C.S., Heinz S., Rosenberg J.L., Perlman E.S., et al. 2004, ApJ, 606,185

Churazov E., Brüggen M., Kaiser C.R., Böhringer H., Forman W., 2001 ApJ 554, 261

Churazov, E., Sunyaev, R., Forman, W., Böhringer, H., 2002, MNRAS, 332, 729

Clemens M.S., Alexander P., Green D.A., 2000, MNRAS 312, 236

Cortese L., Gavazzi G., Boselli A., Iglesias-Paramo J., 2004, A\&A, 416, 119

David, L.P., Forman, W., Jones, C., 1991, ApJ, 380, 39

Della Vecchia C., Bower R., Theuns T., Balogh M., Mazzotta P., Frenk C.S., 2004, MNRAS in press astro-ph/0402441

De Grandi S., Ettori S., Longhetti M., Molendi S., 2004, A\&A 419, 7

De Lucia G., Kauffmann G., White S.D.M., 2004 MNRAS 349, 1101

De Young, D.S., 1978, ApJ, 223, 47

Domainko, W., Gitti, M., Schindler, S., Kapferer, W., 2004a, A\&A 425, L21 
Domainko, W., Kapferer, W., Schindler, S., van Kampen, E., Kimeswenger, S., Ruffert, M., 2004b, Metal enrichment of the intra-cluster medium: ram-pressure stripping of the cluster galaxies, Proceedings of the XXXIXth Rencontres de Moriond on "Exploring the Universe", Y. Giraud-Heraud (ed.), in press, astro-ph/0405577

Domainko, W., Kapferer, W., Schindler, S., van Kampen E., Kimeswenger, S., Ruffert, M., 2004c, Metal enrichment of the intra-cluster medium, Proceedings of Multi-Wavelength Cosmology, Mykonos Island, Plionis M. (ed.)

Domainko, W., Kapferer, W., Schindler, S., van Kampen, E., Kimeswenger, S., Ruffert, M., 2004d, The chemical evolution of the intra-cluster medium, International Astronomical Union, Symposium 217, p.464

Domainko, W., Kapferer, W., Schindler, S., van Kampen E., Kimeswenger, S., Mair M., Kronberger T., Ruffert, M., Mangete O., 2004d, Metal enrichment of the intra-cluster medium: ram-pressure stripping of cluster galaxies, 2004e, submitted to Advances in Space Research

Evrard, A.E., 1991, MNRAS, 248, 8

Fabian A.C. 2004, in: Plasmas in the laboratory and in the universe: new insights and new challenges. AIP Conference Proceedings, 703, 337

Ferrari, C., Maurogordato, S., Cappi, A., \& Benoist, C., 2003, A\&A, 399, 813

Ferrari, C., Benoist, C., Maurogordato, S., Cappi, A., Slezak, E., 2004 A\&A, in press, astroph/0409072

Fujita, Y., Takizawa, M., Nagashima, M., Enoki, M., 1999, PASJ, 51, L1

Fukazawa Y., Makishima K., Tamura T., et al. 1998, PASJ 50, 187

Fukazawa Y., Kawano N., Kawashima K., 2004, ApJ 606, L109

Furusho T., Yamasaki N.Y., Ohashi T., 2003, ApJ 596, 181

Gerhard O., Arnaboldi M. Freeman K.C. Okamura, S., 2002 ApJ, 580 L121

Gnedin, N.Y., 1998, MNRAS, 294, 407

Gunn, J.E., Gott, J.R.III, 1972, ApJ, 176, 1

Hayakawa A., Furusho T., Yamasaki N.Y., Ishida M., Ohashi T., 2004, PASJ in press, astro$\mathrm{ph} / 0409744$

Heinz S., Choi Y., Reynolds C.S. Begelman M.C., 2002, ApJ, 569, 79

Heinz S., Churazov E., Forman W., Jones C., Briel U.G., 2003, MNRAS, 346, 13

Heinz S., 2003, New Astronomy Review, 47, 565

Hidaka M., Sofue Y., 2002, PASJ, 54, 33

Kapferer, W., Breitschwerdt, D., Domainko, W., Schindler, S., van Kampen, E., Kimeswenger, S., 2004a, Metal enrichment and energetics of galactic winds in galaxy clusters, Proceedings of the XXXIXth Rencontres de Moriond on "Exploring the Universe", Y. Giraud-Heraud (ed.), in press, astro-ph/0405576

Kapferer, W., Domainko, W., Schindler, S., van Kampen, E., Kimeswenger, S., Mair M., Kronberger T., Breitschwerdt, D., 2004b, Hydrodynamic galaxy cluster simulations: a challenge for physics, parallel computing and visualisation, submitted to Advances in Space Research

Kenney J.D.P., van Gorkom J.H., Vollmer B., 2004, AJ 127, 3361

Koopmann R., \& Kenney J.D.P. 2004, astro-ph/0406243

Krause M., Camenzind M., 2003, New Astronomy Reviews, 47, 573

Lin Y.-T., Mohr J.J., 2004, ApJ, in press 
Mair M.M., 2004, Numerical Simulations of Galaxy Clusters - Dynamical State and Substructure Analysis, Diploma Thesis, University of Innsbruck

McNamara B.R., Wise M.W., Nulsen P.E.J., David L.P., Carilli C.L., et al. 2001 ApJ 562, 149

Metzler, C.A., Evrard, A.E., 1994, ApJ, 437, 564

Metzler, C.A., Evrard, A.E., 1997, astro-ph/9710324

Moore, B., Katz, N., Lake, G., Dressler, A., \& Oemler, A., 1996, ApJ, 462, 563

Moss, C., Whittle, M., 2000, MNRAS, 317, 667

Nagashima M., Lacey C.G., Baugh C.M., Frenk C.S., Cole S., 2004, astro-ph/0408529

Nulsen P.E.J., David L.P., McNamara B.R., Jones C., Forman W.R., et al. 2002, ApJ 568, 163

Otmianowska-Mazur K., Vollmer B., 2003, A\&A 402, 879

Owen, F.N., Ledlow, M.J., Keel, W.C., Morrison, G.E., 1999, AJ, 118, 633

Peterson J.R., Kahn S.M., Paerels F.B.S., Kaastra J., Tamura T., et al. 2003 ApJ, 590, 207

Quilis, V., Moore, B., Bower, R., 2000, Science, 288, 1617

Rangarajan F.V.N., White D.A., Ebeling H., Fabian A.C., 1995, MNRAS 277, 1047

Ryan-Weber E.V., Meurer G.R., Freeman K.C. et al. 2004, AJ, 127, 1431

Sanders J.S., Fabian A.C., Allen S., Schmidt R.W., 2004, MNRAS 349, 952

Schindler S., 1999, A\&A 349, 435

Schindler, S., Castillo-Morales, A., De Filippis, E., Schwope, A., Wambsganss, J., 2001, A\&A, 376, L27

Schindler, S., Kapferer, W., Domainko, W., van Kampen E., Kimeswenger, S., Ruffert, M., 2004, Evolution of the intra-cluster medium, Proceedings of Multi-Wavelength Cosmology, Mykonos Island, Plionis M. (ed.)

Schmidt R.W., Fabian A.C., Sanders J.S., 2002 MNRAS 337, 71

Schulz S., Struck C., 2001 MNRAS 328, 185

Schumacher E., Hensler G., 2004 IAU Symp. 217, 376

Springel V., Hernquist L., 2003, MNRAS, 339, 289

Tamura T., Kaastra J.S., den Herder J.W.A, Bleeker J.A.M., Peterson J.R., 2004, A\&A 420, 135

Toniazzo T., Schindler S., 2001, MNRAS, 325, 509

Tornatore L., Borgani S., Matteucci F., Recchi S., Tozzi P., 2004, MNRAS 349, L19

Tozzi P., Rosati P., Ettori S., Borgani S., Mainieri V., et al. 2003 ApJ, 593, 705

Valdarnini R., 2003, MNRAS 339, 1117

Veilleux S., Bland-Hawthorn J., Cecil G., Tully R.B., Miller S.T., 1999 ApJ 520, 11

Vollmer B., Cayatte V., Boselli A., Balkowski C., Duschl W.J., 1999, A\&A 349, 411

Vollmer B., Cayatte V., Balkowski C., Duschl W.J., 2001, ApJ 561, 708

Vollmer B., 2003, A\&A 398, 525

Vollmer B., Balkowski C., Cayatte V., van Driel W., Huchtmeier W., 2004a, A\&A 419, 35

Vollmer B., Beck R., Kenney J.D.P., van Gorkom J.H., 2004b, AJ, 127, 3375

Wang, Q.D., Ulmer, M.P., Lavery, R.J., 1997, MNRAS, 288, 702

Zaritzsky D., Gonzales A.H., Zabludoff A.I., 2004, ApJ 613, L93

Zhang H., Koide S., Sakai J., 1999 PASJ 51, 449 